\title{
Tipificación molecular y fenotípica de Staphylococcus aureus resistentes a meticilina (SAMR) en un hospital universitario
}

\author{
Maribel J. Castellano-González, Armindo J. Perozo-Mena, Rosana L. Vivas-Vega, \\ Messaria M. Ginestre-Pérez y Gresleida C. Rincón-Villalobos
}

\section{Molecular and phenotypical typification of methicillin resistant Staphylococcus aureus (MRSA) strains in a university hospital}

Objective: To typify by molecular and phenotypical methods, MRSA strains, isolated from patients and nurses to establish their possible clonal origin. Materials and Methods: 50 MRSA strains isolated in a teaching hospital in Maracaibo (Venezuela) were analyzed. The typification of MRSA strains was performed by means of pulsed-field gel electrophoresis (PFGE) and antibiotyping. Results: In patients, 12 clusters (IXII) and 19 antibiotypes were found; whereas in the health-care personnel, 6 clusters (I-VI) and two antbiotypes were detected. There was no statistically significative association between antibiotypes and band patterns obtained by PFGE $(\mathrm{p}>0.05)$. Conclusions: By means of detection of resistance markers and PFGE, it is feasible to discriminate the nature of the clinical strains of MRSA. The obtained results show the possible nosocomial transmission of MRSA strains and their clonal spread in hospital departments, particularly at the ICU.

Key words: Methicillin-resistant $S$. aureus, pulsed-field gel electrophoresis antibiotypes.

Palabras clave: Staphylococcus aureus resistente a meticilina, electroforesis en gel de campo pulsado, antibiotipia.

\section{Introducción}

$\mathrm{L}$ a aparición de cepas de Staphylococcus aureus resistentes a meticilina (SAMR), fue descrita inicialmente en 1960 , poco tiempo después de la introducción de este antimicrobiano en la práctica clínica. Esta resistencia es conferida por una proteína ligadora de penicilina (sigla en inglés: PBP) adicional conocida como PBP2a o PBP2', la cual no está presente en las cepas susceptibles a meticilina ${ }^{1}$ y es codificada por el gen $m e c A$, el cual forma parte de un complejo móvil (mec) que reside dentro de una isla genómica, un elemento genético denominado cassette cromosómico estafilocóccico (sigla en inglés: SCC) en S. aureus ${ }^{2}$.

Las cepas de SAMR de origen nosocomial pueden transmitirse a la comunidad a través de los pacientes y sus contactos o del personal de salud; a pesar que también existe la probabilidad que estas cepas surjan "de novo", gracias a la adquisición del SCCmec por el genoma de una cepa de $S$. aureus previamente susceptible a meticilina ${ }^{3,4}$.

Por otra parte, ante la aparición simultánea en tiempo y lugar de infecciones causadas por una especie determinada, resulta imprescindible determinar si se trata de la misma cepa en todos los casos, ya que este dato es fundamental para confirmar que se está ante una epidemia o, por el contrario, se trata de cepas diferentes con una coincidencia temporo-espacial casual $^{5}$. En consecuencia, desde el punto de vista epidemiológico, es necesario identificar las fuentes y monitorear la diseminación de las cepas de SAMR durante la investigación de un brote, como parte de un programa de vigilancia y control vigente ${ }^{5}$.

La tipificación, ya sea por métodos fenotípicos o genotípicos, permite determinar si un grupo de cepas de una especie en particular es clonal; es decir, provienen de un precursor común ${ }^{6}$. Este proceso desempeña un importante rol en la comprensión de la epidemiología de SAMR y la evaluación de la efectividad de las medidas de control de las infecciones y prescripción de antimicrobianos ${ }^{7}$.

Diferentes métodos han sido utilizados para la tipificación de SAMR, ${ }^{8}$ entre otros: biotipiaje, antibiogramas $^{9}$ y resistogramas (resistencia a químicos y colorantes), serotipiaje, fagotipia, ribotipia, perfil de plásmidos, proteínas estructurales o enzimo-electro-
Universidad del Zulia, Maracaibo, Venezuela Facultad de Medicina Escuela de Bioanálisis Departamento de Microbiología Cátedra de Bacteriología General (MJC-G, MMG-P)

Cátedra de Práctica Profesional de Bacteriología (AJP-M)

Laboratorio de Bacteriología General y Clínica (RLV-V)

Cátedra de Bacteriología Clínica (GCR-V)

Servicio Autónomo Hospital Universitario de Maracaibo Centro de Referencia Bacteriológíco (AJP-M).

Recibido: 6 de marzo 2008 Aceptado: 28 de noviembre 2008

Fuente de financiamiento.Este proyecto fue financiado por el Consejo de Desarrollo Científico y Humanístico de la Universidad del Zulia (Proyecto No VAC-CONDES 1108-06).

\section{Correspondencia a:} Maribel Josefina CastellanoGonzález maribeljo@cantv.net.ve 
fenotipia, análisis de restricción de ADN cromosómico mediante electroforesis clásica, electroforesis de enzimas multilocus (sigla en inglés: MLEE), electroforesis en gel de campo pulsado (EGCP ó PFGE, según sus siglas en inglés $)^{10,11}$, reacción de polimerasa en cadena $(\mathrm{RPC})^{3}$, hibridación con diferentes sondas (southern blot $)^{12,13}$ o genotipiaje basado en genes de exotoxinas (set) o en el SCCmec ${ }^{2}$.

Desafortunadamente, no todos los métodos clasifican en forma similar; además, pocos estudios han evaluado una amplia gama de aislados comparando simultáneamente múltiples técnicas ${ }^{5}$; no obstante, los resultados de diversas investigaciones sugieren que los marcadores fenotípicos, como biotipos o patrones de susceptibilidad antimicrobiana, poseen limitaciones en su capacidad de tipificación y estabilidad, siendo más propensos a sufrir modificaciones en el tiempo, en comparación a los métodos de genotipificación, como la MLEE y EGCP. Además, muchos de estos sistemas poseen poco poder de discriminación ${ }^{6,13,14}$.

La EGCP, técnica desarrollada por Schwarz y Cantor $^{15}$, ha sido uno de los métodos más utilizados en la epidemiología molecular en los últimos tiempos, siendo considerada actualmente, como el "estandar de oro" para el tipiaje molecular de los organismos ${ }^{16}$. Esta técnica posee un gran poder de discriminación y permite discernir fragmentos de ADN cuya longitud varía de $10 \mathrm{~Kb}$ hasta $7 \mathrm{Mb}$, aproximadamente. Tradicionalmente, los patrones de bandas obtenidos son utilizados para identificar grupos de aislados por similaridad entre patrones. Sin embargo, una debilidad de la EGCP es que los cambios en el patrón de restricción pueden ocurrir en forma aleatoria, ya que se basan en la alteración de un número limitado de sitios en el ADN reconocidos por la enzima de restricción ${ }^{7,17,18}$. Además, ésta es considerada técnicamente compleja, consume mucho tiempo para emitir resultados y los reactivos y/ o equipos a utilizar son sumamente costosos. Es una herramienta útil, no sólo para la tipificación de cepas; sino también, para el seguimiento del desarrollo genético natural de generaciones de $S$. aureus ${ }^{19}$.

La principal ruta de diseminación nosocomial de cepas SAMR entre áreas con diferente localización geográfica, es la propagación de unos pocos clones epidémicos internacionales; aunque el factor determinante del éxito de un clon en su diseminación no ha sido aún dilucidado con claridad; al parecer la presión selectiva de los antimicrobianos podría contribuir a este fenómeno ${ }^{3}$.

Estudios filogenéticos por técnicas moleculares han demostrado que la mayoría de los aislados intrahospitalarios de SAMR pertenecen a pocos linajes o clones que se han diseminado mundialmente y predominan en una región geográfica, a la que, en general, deben su denominación. Al presente, los clones epidémicos reconocidos de mayor diseminación internacional son: Ibérico, Nueva York/Japón, Húngaro, Pediátrico, EMRSA-16 y el clon Brasileño ${ }^{20-23}$.

La determinación de los clones SAMR en América Latina es reciente, encontrándose dos clones predominantes: el clon brasileño y el clon chileno/cordobés ${ }^{24-29}$. No se encontraron reportes en relación a los clones de SAMR predominantes en Venezuela.

Debido a la patogenicidad potencial de las cepas SAMR y a que éstas son aisladas con una frecuencia general de $25,4 \%$ en pacientes $(42,1 \%$ en hospitalizados y $13,9 \%$ en pacientes ambulatorios) y de $16,7 \%$ entre el personal de salud que labora en el hospital (datos no publicados), se consideró pertinente realizar la presente investigación, con el objetivo general de tipificar fenotípicamente (antibiograma) y genotípicamente (EGCP) las cepas de SAMR provenientes de pacientes y personal de salud y determinar el posible origen clonal de las mismas.

\section{Materiales y Métodos}

Cepas bacterianas. En un período de seis meses, se analizaron 286 cepas de $S$. aureus provenientes de pacientes atendidos en los diferentes servicios de un hospital universitario (n: 233) y de personal de enfermería que labora en los diferentes servicios del referido centro de salud (n: 53). La identificación de los aislados fue confirmada utilizando la metodología convencional.

Susceptibilidad antimicrobiana. Fue determinada mediante el método de difusión del disco en agar, siguiendo los lineamientos del Instituto para la Estandarización de Laboratorios Clínicos (CLSI, según sus siglas en inglés $)^{30}$. Se probaron los siguientes antimicrobianos: penicilina $\mathrm{G}(\mathrm{PG} 10 \mathrm{U})$, oxacilina (OX $1 \mu \mathrm{g}$ ), amikacina (AK $20 \mu \mathrm{g}$ ), gentamicina (GM $10 \mu \mathrm{g}$ ), eritromicina (E $15 \mu \mathrm{g})$, clindamicina (CC $2 \mu \mathrm{g}$ ), cloranfenicol (CAF $30 \mu \mathrm{g}$ ), tetraciclina (TE $30 \mu \mathrm{g}$ ), cotrimoxazol (TSX $1,25 / 23,75 \mu \mathrm{g}$ ), rifampicina (RFP $5 \mu \mathrm{g}$ ), vancomicina (VA $30 \mu \mathrm{g}$ ), teicoplanina (TEC $30 \mu \mathrm{g}$ ), ciprofloxacina (CIP $5 \mu \mathrm{g}$ ), levofloxacina (LEV $5 \mu \mathrm{g}$ ), gatifloxacina (GAT $5 \mu \mathrm{g}$ ), linezolid (LZD $30 \mu \mathrm{g}$ ), quinupristina/dalfopristina (QDA $15 \mu \mathrm{g}$ ), mupirocina (MUP $5 \mu \mathrm{g}$ ) y fosfomicina (FOS $50 \mu \mathrm{g}$ ). Las placas inoculadas se incubaron a 35$37{ }^{\circ} \mathrm{C}$ durante 24 horas en atmósfera aeróbica. Para la lectura e interpretación de los resultados de la susceptibilidad a MUP, se adoptaron los criterios de la Sociedad Británica para Antimicrobianos y Quimioterapia $(B S A C)^{31}$ y para FOS, se utilizaron los criterios de interpretación descritos por Gobernado ${ }^{32}$.

Para la antibiotipia, cepas resistentes e intermedias 
fueron tratadas de manera indistinta. Aislados con resistencia, al menos, a un agente antimicrobiano fueron considerados de un antibiotipo distinto a aquellos completamente susceptibles ${ }^{13}$.

Determinación de resistencia a oxacilina. Fenotípicamente, la susceptibilidad antimicrobiana se determinó mediante el método de Kirby \& Bauer, prueba de descarte de resistencia a oxacilina en agar oxacilina $(6 \mu \mathrm{g} / \mathrm{ml})$, determinación de la CIM a oxacilina mediante el método de E-test ${ }^{\circledR}$ y determinación de la PBP2a utilizando la aglutinación de partículas de látex (PBP2a Test OXOID ${ }^{\circledR}$ ).

Preparación del ADN inmovilizado. Las cepas de $S$. aureus fueron crecidas en agar soya tripticasa suplementado con $5 \%$ de sangre de cordero, luego fueron recolectadas y lavadas con una solución $0,5 \%$ $\mathrm{NaCl}$ y $0,01 \mathrm{M}$ de EDTA pH 8,0; posteriormente se mezclaron las células con agarosa de bajo punto de fusión al 1,5\% disuelta en la solución de lavado, utilizando el molde para la fabricación de bloques, se colocó una concentración aproximada de $3 \times 10^{9} \mathrm{UFC} / \mathrm{ml}$ en cada bloque, se prepararon minibloques de $3 \mathrm{~mm}$ x 3 $\mathrm{mm}$ x $0,7 \mathrm{~mm}$ (largo, ancho y grosor). Una vez elaborados los minibloques, se colocaron en solución de lavado conteniendo $10 \mu \mathrm{g} / \mathrm{ml}$ de lisozima, esto se incubó durante 2 horas a $37{ }^{\circ} \mathrm{C}$, posterior a la incubación se eliminó esta solución y se colocaron en una solución desproteinizante que contenía $0,01 \mathrm{M}$ Tris; 0,1M EDTA; $1 \%$ sarcosyl; $1 \%$ nonident $\mathrm{P} 40$ y $4 \mathrm{M}$ urea $\mathrm{pH} 9,5$; los minibloques se incubaron en esta solución por 4 horas a $45^{\circ} \mathrm{C}$. Luego se eliminó esta solución y se lavaron con agua destilada y posteriormente, con una solución $0,01 \mathrm{M}$ Tris-HCL y $0,1 \mathrm{M}$ EDTA $\mathrm{pH} 8$, donde se conservaron a $4^{\circ} \mathrm{C}$ hasta el momento de la digestión.

Digestión del ADN inmovilizado con enzimas de restricción. Cada minibloque fue lavado tres veces en solución $0,01 \mathrm{M}$ Tris-HCL y $0,05 \mathrm{M}$ EDTA pH 8 ; a $4^{\circ} \mathrm{C}$ por 10 minutos, posteriormente se incubaron con $200 \mu \mathrm{l}$ de tampón de digestión de la enzima proporcionado por el fabricante (Promega ${ }^{\circledR}$ ) a $4^{\circ} \mathrm{C}$ por 10 minutos. Posteriormente cada minibloque fue transferido a $100 \mu \mathrm{l}$ de tampón de digestión fresco y se colocaron $20 \mathrm{U}$ de la enzima de restricción SmaI (Promega ${ }^{\circledR}$ ) a cada minibloque, se incubó durante 2 horas a $37^{\circ} \mathrm{C}$, luego se detuvo la reacción reemplazando el tampón por $1 \mathrm{ml}$ de 0,01M Tris-HCL: 0,1M EDTA pH 8,0.

Electroforesis de campo pulsado de los fragmentos de macrorestricción del $A D N$ de $S$. aureus La electroforesis se realizó en una cámara minichef del equipo Guefast $06^{\circledR}$ (NEURONIC, S. A), el gel tenía las siguientes dimensiones $7 \mathrm{~cm}$ x $5 \mathrm{~cm}$ x 0,5 cm (largo, ancho, grosor); se utilizaron $400 \mathrm{ml}$ de tampón TrisBorato EDTA 0,5X (Sigma ${ }^{\circledR}$ ) a $20^{\circ} \mathrm{C}$. Los fragmentos de ADN fueron separados a $10 \mathrm{~V} / \mathrm{cm}$ durante 5 horas en un gel de agarosa al 1,5\% sumergido en el tampón de electroforesis. Los fragmentos de restricción fueron resueltos programando las siguientes rampas con diferentes números de pulsos y tiempos de pulso: 130 pulsos de 21 segundos; 130 pulsos de 17 segundos; 130 pulsos de 13 segundos; 120 pulsos de 9 segundos; 200 pulsos de 5 segundos; 200 pulsos de 3 segundos y 200 pulsos de 1 segundo. El gel se coloreó durante 30 minutos en una solución $0,5 \mu \mathrm{g}$ de bromuro de etidio (Promega ${ }^{\circledR}$ ), luego se visualizó en un transiluminador UV y se fotografió con una cámara digital (SONY DSC707). El tamaño de los fragmentos de restricción en kilopares de bases $(\mathrm{Kb})$, se calculó al comparar la posición de las bandas con la co-migración de un marcador de peso molecular como el fago lambda concatamerizado de $45 \mathrm{~Kb}$ (Promega ${ }^{\circledR}$ ), para bandas de peso molecular pequeño y el genoma completo de Sacharomyces cerevisiae $\left(\operatorname{Biorad}^{\circledR}\right)$, para bandas de peso molecular grande.

Los perfiles obtenidos por EGCP fueron comparados por inspección visual, acorde a los criterios descritos por Tenover ${ }^{14}$, según los cuales, se debe considerar como indistinguibles, aquellas cepas que no presenten diferencias en ninguna de las bandas; cercanamente relacionadas, las que presenten 2 ó 3 fragmentos diferentes; posiblemente relacionadas a las cepas con diferencias en 4 a 6 fragmentos de ADN (bandas); mientras que aquellas con 7 ó más fragmentos diferentes, son consideradas como no relacionadas. Además, se utilizó el análisis computarizado, utilizando el software GUEFASCAN ${ }^{\circledR}$.

Análisis estadístico. Se realizó utilizando el paquete SPSS $^{\mathrm{TM}}$, versión 11.0 (SPSS, Inc, Chicago, III USA) para Windows ${ }^{\mathrm{TM}}$ (Microsoft Corp, Redmond, Washington, USA). En la antibiotipia se utilizó el análisis cluster jerárquico para clasificar las cepas SAMR estudiadas en grupos, de acuerdo a sus semejanzas y diferencias. La asociación de los antibiotipos y patrones de bandas obtenidos por EGCP, se determinó mediante la prueba de Chi-cuadrado, con una significancia del 5\% $(\alpha: 0,05)$.

Control de calidad. Para el control de calidad de las pruebas de tipificación molecular y susceptibilidad antimicrobiana, se utilizaron las cepas $S$. aureus ATCC 29213 (susceptible a OX) y $S$. aureus ATCC 43300 (resistente a OX).

\section{Resultados}

La prevalencia observada para SAMR fue de $17,5 \%$ (50 cepas). De estas, $41(82,0 \%)$ se aislaron a partir de muestras provenientes de pacientes y $9(18,0 \%)$ del personal de enfermería. Al analizar la distribución por 
servicios de las cepas de SAMR aisladas de pacientes, se encontró que 31 aislados $(75,6 \%)$ correspondían a pacientes hospitalizados y $10(24,4 \%)$ a pacientes no hospitalizados. El orden de frecuencia de los aislados según servicio de atención al paciente fue el siguiente: Unidad de Cuidados Intensivos (UCI) 12 (38,7\%), Cirugía 9 (29,0\%), Medicina Interna 4 (12,9\%), Dependencias Externas 4 (12,9\%) y; Neonatología 2 (6,4\%); mientras que la totalidad de cepas SAMR provenientes del personal de enfermería, fueron aisladas en la UCI (Datos no mostrados).

Entre los aislados provenientes de pacientes, todas las cepas SAMR probadas fueron resistentes a dos o más antimicrobianos. Se obtuvieron 19 patrones diferentes de susceptibilidad antimicrobiana, destacando en orden de frecuencia, el perfil 13 que muestra resistencia a 10 antimicrobianos, este fenotipo de resisten- cia fue expresado por 14 cepas de SAMR (34,1\%), seguido del perfil 10 con resistencia a nueve antimicrobianos presente en cuatro cepas $(9,8 \%)$, el perfil 1 con resistencia a dos antimicrobianos (2 cepas, 4,9\%), el perfil 4 con resistencia a cuatro antimicrobianos, presente en dos cepas (4,9\%), el perfil 11 con resistencia a nueve antimicrobianos presente en dos cepas $(4,9 \%)$, los perfiles 14,15 y 16 , con resistencia a 11 antimicrobianos expresados por dos cepas cada uno $(4,9 \%)$ y 1 cepa $(2,4 \%)$ para cada uno de los restantes patrones de resistencia obtenidos (Tabla 1).

En las cepas SAMR aisladas del personal de enfermería se encontraron solamente dos patrones de resistencia, el 1 que presenta resistencia a tres antimicrobianos, expresado por cinco cepas $(55,6 \%) \mathrm{y}$, el 2 con resistencia a 10 antimicrobianos, observado en cuatro cepas $(44,4 \%)$ (Tabla 2$)$.

Tabla 1. Antibiotipos observados en cepas de SAMR aisladas de pacientes 2007. Maracaibo, Venezuela (n: 41)

\begin{tabular}{|c|c|c|c|c|}
\hline Antibiotipo & n antimicrobianos & Antimicrobianos a los que expresa resistencia & n cepas & $\%$ \\
\hline 1 & 2 & PG-OX & 2 & 4,80 \\
\hline 2 & 3 & PG-OX-E & 1 & 2,44 \\
\hline 3 & 3 & PG-OX-CIP & 1 & 2,44 \\
\hline 4 & 4 & PG-OX-E-TEC & 2 & 4,88 \\
\hline 5 & 4 & PG-OX-RFP-TE & 1 & 2,44 \\
\hline 6 & 5 & PG-OX-AK-GM-TSX & 1 & 2,44 \\
\hline 7 & 5 & PG-OX-E-TEC-TE & 1 & 2,44 \\
\hline 8 & 8 & PG-OX-E-CC-AK-GM-CIP-TEC & 1 & 2,44 \\
\hline 9 & 8 & PG-OX-TE-TSX-QDA-TEC-MUP-FOS & 1 & 2,44 \\
\hline 10 & 9 & PG-OX-E-CC-AK-GM-CIP-LEV-TEC & 4 & 9,76 \\
\hline 11 & 9 & PG-OX-E-CC-TE-TSX-TEC-GAT-MUP & 2 & 4,88 \\
\hline 12 & 9 & PG-OX-E-CAF-AK-GM-TSX-CIP-LEV & 1 & 2,44 \\
\hline 13 & 10 & PG-OX-E-CC-AK-GM-CIP-LEV-GAT-TEC & 14 & 34,13 \\
\hline 14 & 11 & PG-OX-E-CC-AK-GM-CIP-LEV-GAT-TEC-QDA & 2 & 4,88 \\
\hline 15 & 11 & PG-OX-E-CC-AK-GM-CAF-CIP-LEV-GAT-TEC & 2 & 4,88 \\
\hline 16 & 11 & PG-OX-E-CC-AK-GM-CIP-LEV-GAT-TEC-FOS & 2 & 4,88 \\
\hline 17 & 12 & PG-OX-E-CC-AK-GM-CIP-LEV-GAT-LZD-QDA-TEC & 1 & 2,44 \\
\hline 18 & 13 & PG-OX-E-CC-GM-CAF-CIP-LEV-GAT-TEC-MUP-TE-TSX & 1 & 2,44 \\
\hline 19 & 13 & PG-OX-E-CC-TE-TSX-CIP-LEV-LZD-QDA-TEC-MUP-FOS & 1 & 2,44 \\
\hline
\end{tabular}

Tabla 2. Antibiotipos encontrados en cepas de SAMR aisladas en personal de enfermería 2007. Maracaibo, Venezuela (n: 9)

\section{Antibiotipo}

1

2 n antimicrobianos

3

10
Antimicrobianos a los que expresa resistencia

PG-OX-TE

PG-OX-E-CC-AK-GM-CIP-LEV-GAT-TEL n cepas

5

4
$\%$

55,56

44,44 
El análisis de los productos de restricción obtenidos al digerir el genoma de las cepas de SAMR con SmaI, permitió identificar doce patrones diferentes de bandas, revelando la presencia de, al menos cinco grupos, designados con números romanos (I-V). Cada uno de los restantes patrones se presentó en forma aislada y fueron también designados con números romanos (VI-XII). El grado de semejanza entre las cepas se representó gráficamente como un dendrograma de homología (Figura 1).

Los fenotipos de resistencia (antibiotipos) encontrados en las cepas de pacientes y su relación con los grupos obtenidos mediante EGCP se presentan en la Tabla 3. Así, el grupo I estaba formado por tres cepas del antibiotipo 13 y una del 16. El grupo II estaba compuesto por dos cepas del antibiotipo 13, una del 10 y una del 15. El grupo III estuvo integrado por cuatro cepas con el fenotipo de resistencia 13, dos con el 12, una con el 10 y una con el 14. El IV, presentó una cepa del antibiotipo 2 y una del 11; mientras que el V mostró una cepa de cada uno de los siguientes antibiotipos: $10,13,15,16,18$ y 19. De los grupos restantes, cuatro cepas expresaron el antibiotipo 13, dos el 4 y una el 6 .

Entre las cepas provenientes de personal de enfer-

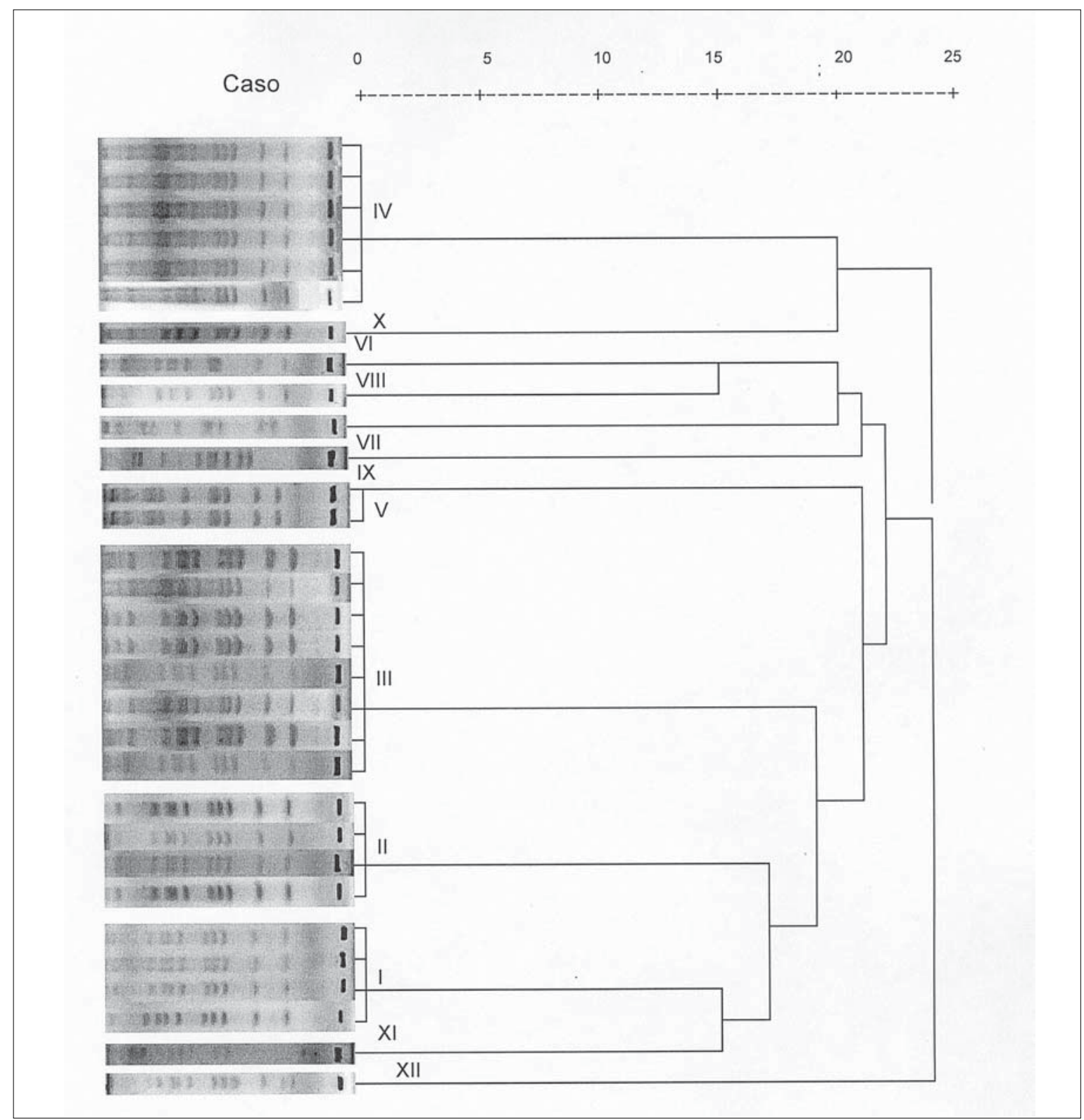

Figura 1. Dendograma derivado del análisis de la EGCP en cepas de SAMR aisladas de pacientes. 2007. Maracaibo, Venezuela (n: 31). 
Tabla 3. Antibiotipos en cepas de SAMR aisladas de pacientes según grupos obtenidos mediante EGCP. 2007. Maracaibo, Venezuela ( $n$ : 31)

\begin{tabular}{|c|c|c|c|}
\hline Grupo & Antibiotipo & n cepas & $\%$ \\
\hline \multirow[t]{2}{*}{ I } & 13 & 3 & 75,0 \\
\hline & 16 & 1 & 25,0 \\
\hline \multirow[t]{3}{*}{11} & 10 & 1 & 25,0 \\
\hline & 13 & 2 & 50,0 \\
\hline & 15 & 1 & 25,0 \\
\hline \multirow[t]{4}{*}{ III } & 10 & 1 & 12,5 \\
\hline & 12 & 2 & 25,0 \\
\hline & 13 & 4 & 50,0 \\
\hline & 14 & 1 & 12,5 \\
\hline \multirow[t]{2}{*}{ IV } & 2 & 1 & 50,0 \\
\hline & 11 & 1 & 50,0 \\
\hline \multirow[t]{6}{*}{ v } & 10 & 1 & 16,6 \\
\hline & 13 & 1 & 16,6 \\
\hline & 15 & 1 & 16,6 \\
\hline & 16 & 1 & 16,6 \\
\hline & 18 & 1 & 16,6 \\
\hline & 19 & 1 & 16,6 \\
\hline VI & 13 & 1 & 100 \\
\hline VII & 4 & 1 & 100 \\
\hline VIII & 13 & 1 & 100 \\
\hline IX & 13 & 1 & 100 \\
\hline$x$ & 6 & 1 & 100 \\
\hline$X I$ & 4 & 1 & 100 \\
\hline XII & 13 & 1 & 100 \\
\hline
\end{tabular}

mería, con SmaI, se identificaron seis patrones diferentes de bandas, identificados con los números romanos I al VI, respectivamente. El grado de semejanza entre las cepas de SAMR aisladas del personal de enfermería, se representó gráficamente como un dendrograma de homología (Figura 2). En el mismo puede evidenciarse la presencia de al menos un grupo, designado con el número I, el cual estaba formado por cuatro cepas, aisladas de una misma persona; mientras que el resto de los aislados se presentaron en forma única y fueron designados también con números romanos (II al VI, respectivamente).

Los fenotipos de resistencia (antibiotipos) encontrados en las cepas de SAMR aisladas de personal de enfermería y su relación con los grupos obtenidos mediante EGCP se presentan en la Tabla 4. Así, el grupo I estaba formado por cuatro cepas del antibiotipo 2; mientras que cada una de las restantes cepas únicas, mostraron el fenotipo de resistencia 1.

Al relacionar los grupos obtenidos de las cepas de SAMR aisladas de pacientes y personal de enfermería, pudo evidenciarse, que el grupo I que portaba un miembro del personal de salud, correpondió al grupo III aislado de pacientes, expresado por ocho cepas, cuatro de las cuales provenían de la UCI y expresaron el mismo patrón de resistencia.
Figura 2. Dendograma derivado del análisis de la EGCP en cepas de SAMR aisladas en personal de enfermería. 2007. Maracaibo, Venezuela (n: 9).

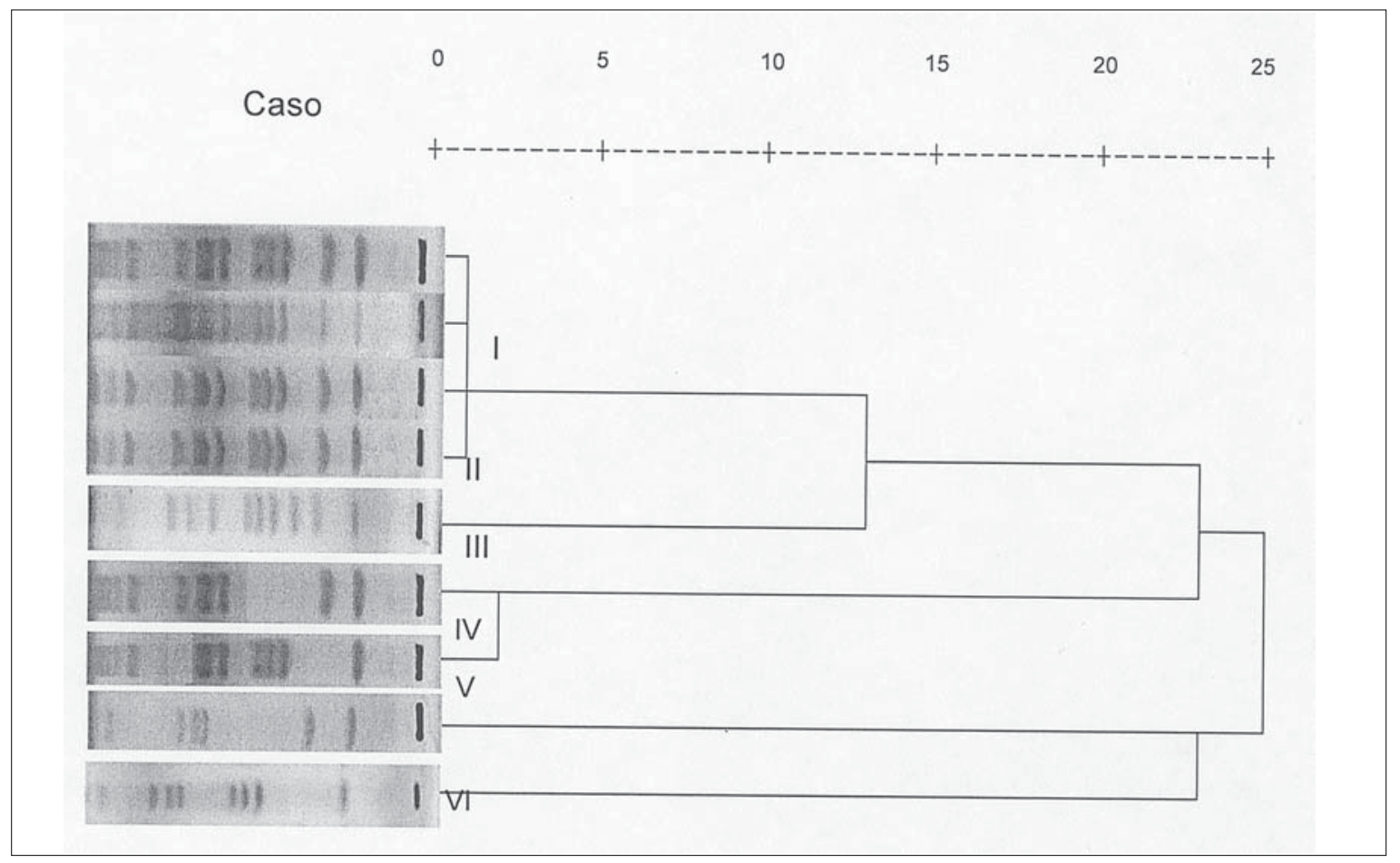




\section{Discusión}

Los resultados obtenidos muestran claramente la diseminación policlonal de los aislados de SAMR en los diferentes servicios de atención al paciente en el hospital; con excepción de la UCI, donde, por ambos métodos de tipificación, se encontró transmisión intrahospitalaria del microorganismo y se demostró la diseminación clonal de las cepas estudiadas.

La antibiotipia es uno de los métodos más sencillos y económicos que pueden ser utilizados en el laboratorio clínico (particularmente, en los pequeños) para la diferenciación de cepas bacterianas ${ }^{33}$; pero sólo cuando los diámetros de las zonas de inhibición son utilizados como marcadores y, no las interpretaciones categóricas ${ }^{5}$, ya que diferentes investigaciones han demostrado que los antibiogramas basados en categorías de interpretación suelen ser problemáticos ${ }^{8,33,34}$. Cambios en los tamaños de los halos de inhibición para un solo agente no son significativos; por lo tanto, antes de considerar como diferentes a dos cepas deben observarse modificaciones en los halos de dos o más antibióticos 5 . Esta inestabilidad en los patrones de resistencia antimicrobiana en $S$. aureus, probablemente está, al menos en parte, relacionada con cambios en el contenido de plásmidos ${ }^{5}$.

Los patrones de multiresistencia exhibidos por la mayoría de las cepas de SAMR intrahospitalarias han asistido por muchos años a los microbiólogos clínicos en la discriminación entre cepas de $S$. aureus mecA positivas y negativas; sin embargo, las variaciones en los perfiles de susceptibilidad y el incremento general en la frecuencia de aislamiento de cepas de SAMR no multi-resistentes ha dificultado la interpretación fenotípica ${ }^{34}$.

La determinación de 19 patrones diferentes de resistencia antimicrobiana (antibiotipos) entre las cepas aisladas de pacientes y dos en las del personal de enfermería, permitió analizar, el posible grado de asociación entre las cepas, que es aparentemente bajo. A excepción del patrón 13 , el bajo número de cepas coincidentes en un mismo patrón de resistencia, indica que carecen de un origen común (al menos recientemente), ya que no responden igual frente a los antimicrobianos.

De los resultados mostrados en las Tablas 1 y 2 , es evidente que 38 cepas ( 34 para $89,5 \%$ del total de cepas probadas de pacientes y $4(80,0 \%)$ del personal de salud), presentaron multirresistencia importante frente a cinco o más antimicrobianos. Esto constituye una complicación desde el punto de vista del manejo terapéutico adecuado para tratar a los pacientes afectados de infecciones por estas cepas, así como controlar su diseminación y evitar brotes de infecciones nosocomiales $^{35}$
Tabla 4. Antibiotipos en cepas de SAMR aisladas en personal de enfermería, según grupos obtenidos mediante EGCP. 2007. Maracaibo, Venezuela (n: 9)

\begin{tabular}{|cccc|}
\hline Grupo & Antibiotipo & n cepas & $\%$ \\
\hline I & 2 & 4 & 100 \\
II & 1 & 1 & 100 \\
III & 1 & 1 & 100 \\
IV & 1 & 1 & 100 \\
\hline$V$ & 1 & 1 & 100 \\
\hline$V I$ & 1 & 1 & 100 \\
\hline
\end{tabular}

El patrón 1 presentado por cinco cepas del personal de enfermería es similar al patrón 2 observado entre las cepas aisladas de pacientes, además, el patrón de susceptibilidad antimicrobiana 13 de los pacientes, es similar al 2, presentado por cuatro de las cepas recuperadas del personal $\mathrm{y}$, prácticamente, incluye los patrones previos, observados entre pacientes, reforzando las afirmaciones de diferentes autores, según los cuales, el personal de salud está en riesgo de ser colonizado con cepas potencialmente más resistentes a los antimicrobianos y diseminar el organismo a pacientes mediante contacto directo ${ }^{35}$.

Un hallazgo importante de este trabajo es que los perfiles de susceptibilidad antimicrobiana entre cepas provenientes de personal de enfermería y pacientes, son diferentes en ocho de 17 antimicrobianos ( $\sin$ incluir a PG y OX), permitiendo considerarlos como dos variedades diferentes. Sin embargo, podría afirmarse que el personal de salud constituye un reservorio importante de los SAMR que colonizan a pacientes del mismo servicio ya que al identificar el personal de salud como vector de transmisión, sus cepas reflejan los perfiles de resistencia prevalentes en el hospital ${ }^{5,35}$.

En este caso, los resultados obtenidos muestran en general, mayores porcentajes de resistencia en las cepas provenientes de pacientes en comparación con los aislados del personal de enfermería, lo cual podría deberse a que durante la permanencia de los pacientes en el hospital existe mayor probabilidad de ser colonizado o infectado por cepas bacterianas más resistentes a los antimicrobianos. La presión selectiva natural juega un importante papel predominante en el medio hospitalario, donde el uso de los antimicrobianos es frecuente, favoreciendo la aparición y diseminación de cepas más resistentes.

Se podría sugerir que algunos de los patrones de multiresistencia presentes en las cepas de S. aureus, se encuentran conferidos por plásmidos. De este modo, si los genes que confieren resistencia a múltiples antimicrobianos se encuentran ligados en el mismo plásmido, la administración de un solo antibacteriano 
conduciría, de manera indirecta, a la selección de cepas resistentes al resto de los antimicrobianos ${ }^{31}$. La coincidencia entre 14 cepas provenientes de pacientes en el patrón 13 correspondiente a resistencia frente a 10 antimicrobianos, cuyos orígenes de aislamiento son: 10 del servicio de la UCI, dos de Cirugía y dos del servicio de Medicina Interna, permite establecer que aparentemente están relacionadas.

Del total de cepas SAMR estudiadas en pacientes (n: 41) sólo se realizó EGCP a 31. La diferencia observada en el número de cepas, se debe a que diez de éstas provenían de pacientes no hospitalizados o referidos de otros centros hospitalarios, por lo que no fue posible efectuar el seguimiento epidemiológico de las mismas. Por su parte, la totalidad de cepas de SAMR aisladas del personal fueron analizadas por EGCP (n: 9).

Entre los pacientes, el dendrograma indica la presencia de más de 10 pequeños grupos, resultados similares a los descritos en Japón ${ }^{37}$, lo que sugiere que las infecciones por SAMR fueron provocadas por cepas de diferentes genotipos, a diferencia de las cepas provenientes del personal de enfermería, que sólo permitieron la obtención de un pequeño grupo, sugiriendo que la colonización del personal de la UCI era de origen clonal.

Por otra parte, la detección de cepas con patrón de resistencia o perfil molecular idéntico, es indicativo de diseminación clonal; lo más importante en este caso es la implementación de medidas de barrera y lavado de manos, así como el aislamiento de los pacientes índi$\operatorname{ces}^{38}$. Por otro lado, cuando en un grupo de cepas resistentes se encuentra una gran variedad genética, eso nos indica que está ocurriendo una selección independiente de la resistencia por presión selectiva; en este caso, se debe cambiar la política de uso de antimicrobianos para intentar seleccionar menos ese tipo de resistencia.

Las cepas SAMR tienden a ser similares en muchas características fenotípicas y al parecer pertenecen a una subpoblación evolutivamente restringida dentro de la especie $\mathrm{e}^{39-42}$. Como resultado, la discriminación entre estas cepas por métodos fenotípicos, como el antibiograma, es frecuentemente complicada $33,39,42-44$.

En forma similar a los reportes de Kawano en Japón ${ }^{45,46}$, a pesar que, estadísticamente, no pudo demostrarse relación entre los resultados de los dos métodos ( $p>0,05)$, en general hubo buena concordancia entre los resultados de la tipificación molecular (EGCP) y los fenotípicos (antibiotipia): cepas dentro de un mismo grupo tienden a expresar el mismo fenotipo de resistencia antimicrobiana. No obstante, cepas de un mismo grupo pueden presentar fenotipos diferentes y viceversa, un mismo antibiotipo puede estar presente en dos o más grupos. Según Yoshida ${ }^{47}$, el análisis de grupos basado en patrones de susceptibilidad antimicrobiana (antibiotipia) brinda resultados similares a la EGCP en $79 \%$ de los casos. De la misma manera, según los reportes de Struelens ${ }^{13}$, las variaciones en los patrones de resistencia antimicrobiana con respecto a las cepas prototipo, al parecer no son relevantes desde el punto de vista epidemiológico, cuando éstas son disociadas de las variaciones observadas en los perfiles genéticos. Estos hallazgos sugieren que la inestabilidad fenotípica in vivo o in vitro puede afectar la reproducibilidad más que el poder discriminante de los métodos de tipificación ${ }^{13,48}$.

Las discrepancias observadas entre ambos métodos de tipiaje sugieren que la variación fenotípica dentro de los tipos obtenidos por métodos moleculares podría representar artefactos in vitro. El hecho de si estas variaciones fenotípicas realmente son el reflejo de cambios en el ADN plasmídico o constituyen artefactos, debe ser clarificado antes de recomendar la combinación de ambos métodos para incrementar el poder de discriminación de las técnicas ${ }^{42}$.

De particular interés fue la observación de que la relación genética de las cepas, determinada por el análisis de los patrones de macro-restricción, parece correlacionar con la distancia respectiva en lugar y tiempo de los aislados. Esta observación podría sugerir que la determinación de la similitud entre los patrones de bandas es proporcional a la conservación de la distribución de los sitios de restricción en el cromosoma bacteriano $^{13,37}$. Esta concordancia, aunada a la coincidencia de las cepas en tiempo y espacio, sugiere la ocurrencia de un pequeño brote por SAMR en las instalaciones de la UCI del hospital durante el período estudiado.

La variedad de patrones obtenidos por EGCP demuestra un patrón multifocal de expansión de SAMR en este hospital, a excepción de la UCI. Estos resultados son similares a los publicados por Sasaki R y $\operatorname{cols}^{49}$ y podrían obedecer al hecho que una vez que el paciente recluido en la UCI mejora su condición es trasladado a otros servicios clínicos, dependiendo de la disponibilidad de camas, permitiendo así, la diseminación de cepas de SAMR entre los diferentes servicios.

En conclusión puede decirse que, por ambos métodos, sólo fue posible determinar transmisión intrahospitalaria de cepas de SAMR en la UCI del hospital. La resistencia a los antimicrobianos en los patrones estudiados fue heterogénea. No se encontró asociación estadísticamente significativa entre la resistencia a los antimicrobianos y los patrones de la EGCP. Los resultados obtenidos en este trabajo son de gran importancia epidemiológica y demuestran la diseminación clonal 
de SAMR en las instalaciones del hospital, particularmente en la UCI, durante el período estudiado.

Agradecimiento. Al Consejo de Desarrollo Científico y Humanístico de la Universidad del Zulia por el financiamiento de esta investigación. Al Profesor Ramón Pérez por su asesoría en el análisis estadístico de los resultados.

\section{Resumen}

Objetivo: Tipificar por métodos moleculares y fenotípicos, las cepas SAMR aisladas de pacientes y personal de enfermería para establecer su posible origen clonal. Materiales y Métodos: Se analizaron 50 cepas SAMR aisladas en un Hospital Universitario de
Maracaibo (Venezuela). La tipificación se efectuó mediante electroforesis en gel de campo pulsado (EGCP) y antibiotipia. Resultados: Entre pacientes, se obtuvieron 19 antibiotipos y 12 grupos (I-XII); mientras que, en el personal de salud, por EGCP se detectaron seis grupos (I-VI) y dos antibiotipos. No se encontró asociación estadísticamente significativa entre los antibiotipos y patrones de bandas obtenidos por EGCP $(p>0,05)$. Conclusiones: Por medio de la detección de marcadores de resistencia y mediante la EGCP, es factible diferenciar la naturaleza de las cepas SAMR de origen clínico. Los resultados obtenidos demuestran la posible transmisión intrahospitalaria de cepas SAMR; así como, su diseminación clonal en los servicios del hospital, particularmente en la UCI, durante el período estudiado.

\section{Referencias}

1.- Bannerman T. Staphylococcus, Micrococcus, and other catalase-positive cocci that grow aerobically. En Murray P, Baron E, Jorgensen J, Pfaller M, Yolken R. Manual of Clinical Microbiology. $9^{\text {th }}$ Edition. Volumen 1. Chapter 28. ASM Press. Washington, D. C. USA. 2007. pp: 390-411.

2.- Chongtrakool P, Ho T, Xue X, Kondo Y, Trakulsomboon S, Tiensasitorn C. et al. Staphylococcal cassette chromosome mec (SCCmec) typing of methicillin-resistant Staphylococcus aureus strains isolated in 11 Asian countries: a proposal for a new nomenclature for SCCmec elements. Antimicrob. Agents Chemother 2006; 50: 1001-12.

3.- Nour M, Mastouri M, Ben Nejma M. Methicillin resistance in Staphylococcus aureus; emergence and molecular basis Pathol Biol (París) 2005; 53: 334-40.

4.- Durand G, Bes M, Meugnier H, Enright M, Forey F, Liassine N. et al. Detection of new methicillin-resistant Staphyloccus aureus clones containing the toxic shock syndrome toxin 1 gene, responsible for hospital-andcommunity-acquired infections in France. $\mathrm{J}$ Clin Microbiol 2006; 44: 847-53.

5.- Kim Y K, Kim J S, Kim H S, Song W, Cho H C, Lee K M. Molecular typing of Staphylococcus aureus isolated from blood on the basis of coagulase gene polymorphism and toxin genes. Korean $\mathrm{J}$ Lab Med 2008; 28: 286-90.

6.- Blumberg H, Rimland D, Kiehlbauch J, Terry P M, Wachsmuth I. Epidemiologic typing of Staphylococcus aureus by DNA restriction fragment length polymorphisms of rRNA genes: elucidation of the clonal nature of a group of bacteriophage-non typeable, ciprofloxacin-resistant, methicillin-susceptible $S$. aureus isolates. J Clin Microbiol 1992; 30: 362-9.

7.- Murchan S, Kauffmann M E, Deplano A, De Rycck R, Struellens M, Elsberg Ch. Harmonization of pulsed-field electrophoresis protocols for epidemiological typing of strains of methicillin-resistant Staphylococcus aureus. a single approach develop by consensus in 10 european laboratories and its application for tracing the spread of related strains. J Clin Microbiol 2003; 41: 1574 -85.

8.- Archer G, Maryhall C. Comparison of epidemiologic markers used in the investigation of an outbreak of methicillin-resistant Staphylococcus aureus infection. J Clin Microbiol 1983; 1: $395-9$.

9.- Aparicio G, Paz-Ramírez M, Ribas-Aparicio R, Giono-Cerezo S, Rivera E. Resistencia a antibióticos por Staphylococcus aureus de origen diverso y su utilidad en la tipificación de cepas. Rev Lab Acta1994; 6: 47-52.

10.- Duck W, Steward C, Banerjee S, McGowan J, Tenover F. Optimization of computer software setting improves accuracy of pulsed-field electrophoresis macrorestriction fragment pattern analysis. J Clin Microbiol 2003; 41: 3035-42.

11.- McDougal LK, Steward CD, Killgore GE, Chaitram JM, McAllister S, Tenover FC. Pulsed-field electrophoresis typing of oxacillin-resistant Staphylococcus aureus isolates from the United States: establishing a national database. J Clin Microbiol 2003; 41: 5113-20.

12.- Aguiar-Alves F, Medeiros F, Fernándes O, Gudziki R, Perdreaw-Remington F, Riley L.
New Staphylococcus aureus genotyping method based on exotoxin (set) genes. J Clin Microbiol 2006; 44: 2728-32.

13.- Struelens M, Deplano A, Godard C, Maes N, Serrys E. Epidemiologic typing and delineation of genetic relatedness of methicillin-resistant Staphylococcus aureus by macrorestriction analysis of genomic DNA by using pulsed-field electrophoresis. J Clin Microbiol 1992; 30: 2599-2605.

14.- Tenover F, Arbeit R, Goering R, Mickelsen P, Murray B, Persing D, et al. Interpreting chromosomal DNA restriction patterns produced by pulsed-field gel electrophoresis: Criteria for bacterial strain typing. J Clin Microbiol 1995; 33: 2233-9.

15.- Schwarz D, Cantor C. Separation of yeast chromosome-sized DNAs by pulsed-field gradient gel electrophoresis. Cell 1984; 7: 664-74.

16.- Birren B, Lai E. Pulsed field gel electrophoresis: a practical guide. San Diego. USA. Academic Press. 1993. pp: 1-100.

17. - Mulvey M, Chui I, Ismail J, Louie L, Murphy C, Chang N. et al. Development of a Canadian standardized protocol for subtyping methicillin-resistant Staphylococcus aureus by using pulsed-field gel electrophoresis. J Clin Microbiol 2001; 39: 3481-5.

18.- Stranden A, Frei R, Widmer A. Molecular typing of methicillin-resistant Staphylococcus aureus: can PCR replace pulsed-field gel electrophoresis? J Clin Microbiol 2003; 41: 381-6.

19. - Schlichting C, Branger C, Fournier J, Witte W, Boutonnier A, Woez C. et al. Typing of Staphylococcus aureus by pulsedfield electrophoresis, zymotyping, capsule typing and phage typing resolution of clonal 
relationships. J Clin Microbiol 1993; 31: 227-32.

20.- Nabón A. Staphylococcus aureus resistente a betalactámicos en infecciones detectadas en la comunidad. Salud Militar 2006; 28: 26-33.

21.- Aires de Sousa M, Miragaia M, Santos I, Avila S, Adamson I, Casagrande S, et al. Three-year assesment of methicillinresistant Staphylococcus aureus clones in Latin America from 1996 to 1998. J Clin Microbiol 2001; 39: 2197-205.

22.- Amorim M, Faria N, Oliveira D, Vasconcelos C, Cabeda J, Mendes A, et al. Changes in the clonal nature and antibiotic resistance profiles of methicillin-resistant Staphylococcus aureus isolates associated with spread of the EMRSA-15 clone in a tertiary care Portuguese hospital. J Clin Microbiol 2007; 45(9): 2881-8.

23.- Aires de Sousa M, Bartzavali C, Spiliopoulou I, Sánches I, Crisóstomo M, de Lencastre H. Two international methicillin-resistant Staphylococcus aureus clones endemic in a university hospital in Patras, Greece. J Clin Microbiol 2007; 41 (5): 2027-32.

24.- Bustos-Martínez J, Handam-Partida A, Gutiérrez-Cárdenas M. Staphylococcus aureus: la re-emergencia de un patógeno en la comunidad. Rev Biomed 2006; 17: 285-305.

25.- Noriega L, González P, Hormazabal J, Pinto C, Canals M, Munita J. Staphylococcus aureus comunitario resistente a cloxacilina. Comunicación de los primeros cinco casos descritos en Chile. Rev Méd Chil 2008; 136: 885-91.

26.- Galiana A. Infección por Staphylococcus aureus meticilino resistente adquirido en la comunidad. Arch Pediatr Urug 2003; 74(1): 26-9.

27.- Ribeiro A, Dias C, Silva C, Berquó L, Antunes F, Soares R. et al. Firts report of infection with community acquired methicillin-resistant Staphylococcus aureus in South America. J Clin Microbiol 2005; 43: 1985-8.

28. - Palombarani S, Gardella N, Tuduri A, Figuera S, Sly G, Corazza R, et al. Infecciones adquiridas en la comunidad por Staphylococcus aureus resistente a meticilina en un hospital de agudos. Rev Argent Microbiol 2007; 39: 151-5.

29. - Gardella N, Picasso R, Pedrani S, Lasala M, Foccoli M, Benchetrit G, et al. Methicillinresistant Staphylococcus aureus strains in Buenos Aires teaching hospitals: replacement of multidrug resistant South
American clone by another susceptible to rifampicin, minocyclin and trimethoprim/ sulfamethoxazole. Rev Argent Microbiol 2005; 37: 156-60.

30.- CLSI (Clinical Laboratory Standardization Institute). Antimicrobial Susceptibility

Testing. Eighteenth Informational

Supplement. Document M100/S18 Vol: 28 $\mathrm{N}^{\circ}$ 1. 2008; pp 46-52; 110-14.

31.- BSAC- British Society for Antimicrobial and Chemotherapy. 2003. Disc diffusion method of antimicrobial susceptibility testing. Version 2.1.4. 2003. pp: 1-50.

32.- Gobernado M. Fosfomicina. Rev Esp Quimioterap 2003; 16: 15-40.

33. - Shittu A O, Lin J. Antimicrobial susceptibility patterns and characterization of clinical isolates of Staphylococcus aureus in KwaZulu-Natal Province, South Africa. BMC Infect Dis 2006; 6: 125-38.

34. - Merlino J, Watson J, Rose B, Beard-Pegler M, Gottlieb T, Bradburry R, et al. Detection and expression of methicillin/oxacillin resistance in multi-drugresistant and non- multi-drug-resistant Staphylococcus aureus in Central Sidney, Australia. J Antimicrob Chemother 2002; 49: 793-801.

35.- Céspedes C, Miller M, Quagliarello B, Vavagiakis P, Klein R, Lowy F. Differences between Staphylococcus aureus isolates from medical and non-medical hospital personnel. J Clin Microbiol 2002; 40: 2594-7.

36.- Paniagua G, Monroy E, Vaca S, González S. Resistencia a antibióticos y metales pesados en cepas de Staphylococcus aureus. Rev Med Hosp Gen Mex 2003; 66: 13-21.

37.- Kaneiko K, Iwai A, Saruta K, Fujino T, Nakamura A, Hamada Y. et al. Molecular epidemiology of a methicillin-resistant Staphylococcus aureus infection. Jpn J Infect Dis 2000; 53: 242-3.

38.- Sader H. Resistencia antimicrobiana en Latinoamérica ¿Cómo estamos? Rev Chil Infect 2002; 19(Suppl 1): S5-S13.

39.- Crisóstomo M I, Westh H, Tomasz A, Chung M, Oliveira D C, de Lencastre H. The evolution of methicillin resistance in Staphylococcus aureus: similarity of genetic backgrounds in historically early methicillinsusceptible and -resistant isolates and contemporary epidemic clones. Proc Natl Acad Sci U S A 2001; 98: 9865-70.

40.- Oliveira D C, Tomasz A, de Lencastre H. The evolution of pandemic clones of methicillin-resistant Staphylococcus aureus: identification of two ancestral genetic backgrounds and the associated mec elements. Microb Drug Resist 2001; 7: 349-61.

41.- McAleese F, Murphy E, Babinchak T, Singh G, Said-Salim B, Kreiswirth B, et al. Use of ribotyping to retrospectively identify methicillin-resistant Staphylococcus aureus isolates from phase 3 clinical trials for tigecycline that are genotypically related to community-associated isolates. Antimicrob Agents Chemother 2005; 49: 4521-9.

42.- Ostojiæ M. Epidemiologic genotyping of methicillin-resistant Staphylococcus aureus (MRSA) by pulsed-field gel electrophoresis (PFGE). Bosn J Basic Med Sci 2008; 8: 259-65.

43.- Wolter D J, Chatterjee A, Varman M, Goering $\mathrm{R}$ V. Isolation and characterization of an epidemic methicillin-resistant Staphylococcus aureus 15 variant in the central United States J Clin Microbiol 2008; 46: 3548-9.

44.- Fenner L, Widmer A F, Dangel M, Frei R. Distribution of spa types among meticillinresistant Staphylococcus aureus isolates during a 6 year period at a low-prevalence University Hospital. J Med Microbiol 2008; 57: 612-16.

45.- Kawano F, Miyazaki H, Takami J, Fujino T, Sekiguchi J, Saruta K, et al. Molecular epidemiology of methicillin-resistant Staphylococcus aureus in a Kumamoto hospital in 2002. Jpn J Infect Dis 2002; 56: 129-32.

46.- Kawano F, Miyazaki H, Takami J, Fujino T, Sekiguchi J. Molecular epidemiology of methicillin-resistant Staphylococcus aureus in Kumamoto hospital in 2002. Jpn J Infect Dis 2003; 56: 129-31.

47.- Yoshida J, Ishimaru T, Kawano S, Akao M. An electronic system combining MIC2000TM and antibiogram cluster analysis for surveillance of methicillinresistant Staphylooccus aureus in hospitals. Jpn J Infect Dis 2000; 53: 1-5.

48.- Vivoni A, Diep B, De Gouveia A, Santos K, Riley L, Sensabaugh G, et al. Clonal composition of Staphylococcus aureus isolates at a Brazilian university hospital: Identification of international circulating lineages. J Clin Microbiol 2006; 44: 168691.

49.- Sasaki R, Fujino T, Saruta K, Kawasaki J, Shigea N, Kirikae T. Molecular epidemiological surveillance of methicillin-resistant Staphylococcus aureus in a Hiroshima community hospital in 2002. Jpn J Infect Dis 2002; 55: $93-5$. 